\title{
Diversité culturelle et plurilinguisme en Asie du
} Sud-Est

L'exemple de Singapour

Pierre-Louis Gauthier

\section{CpenEdition}

\section{Journals}

Édition électronique

URL : http://journals.openedition.org/ries/2949

DOI : 10.4000/ries.2949

ISSN : 2261-4265

Éditeur

Centre international d'études pédagogiques

Édition imprimée

Date de publication : 1 mars 1998

Pagination : 61-68

ISBN : 9771254459005

ISSN : 1254-4590

\section{Référence électronique}

Pierre-Louis Gauthier, "Diversité culturelle et plurilinguisme en Asie du Sud-Est », Revue internationale d'éducation de Sèvres [En ligne], 17| 1998, mis en ligne le 19 avril 2013, consulté le 10 décembre 2020 URL : http://journals.openedition.org/ries/2949; DOI : https://doi.org/10.4000/ries.2949

Ce document a été généré automatiquement le 10 décembre 2020.

(c) Tous droits réservés 


\section{Diversité culturelle et plurilinguisme en Asie du Sud-Est}

L'exemple de Singapour

Pierre-Louis Gauthier

The school will do its utmost best to enable the pupils to be effectively bilingual. It will inculcate in pupils moral and cultural values consistent with a multi-racial, multi-religious and multi-cultural modem Singapore. Yumin Primary School's School Policy

1 Depuis plus d'une décennie, Singapour, l'un des quatre dragons de l'Asie du Sud-Est, affiche une prospérité insolente au regard des pays de la zone aussi bien que des grands ensembles mondiaux.

2 Forte de cette situation, la cité-État se classe dans le groupe des pays très puissants, avec un niveau de vie qui dépasse celui de la France, de la Suède, du Canada, et même, ô ironie de l'histoire, celui de l'ancien colonisateur, le Royaume-Uni.

3 Cette indéniable réussite s'opère sur un fond de société multiraciale et s'appuie en grande part sur un système d'éducation et de formation qui a su non seulement intégrer la dimension multiculturelle et plurilinguistique, mais en faire également un atout socioéconomique positif. 


\begin{tabular}{|c|c|}
\hline \multicolumn{2}{|c|}{$\begin{array}{c}\text { Fiche d'identité } \\
\text { LA RÉPUBLIQUE DE SINGAPOUR }\end{array}$} \\
\hline Superficie : $620 \mathrm{~km} 2$ & France : $549000 \mathrm{~km} 2$ \\
\hline Population : 3000000 hab. & France : 58200000 hab. \\
\hline Accroissement moyen population /an : 54000 hab. $(+2 \%)$ & France : 350000 hab. $(+6 \%)$ \\
\hline Population prévue en $2025: 4000000$ ( $+33 \%$ en 30 ans) & France : 63,6 millions ( $+9 \%$ en 30 ans) \\
\hline Densité absolue : $4840 \mathrm{hab} / \mathrm{km} 2$ & France : $106 \mathrm{hab} . / \mathrm{km}^{2}$ \\
\hline Capitale : Singapour (3 800000 hab.) & France : Paris ( 9500000 hab.) \\
\hline \multicolumn{2}{|l|}{ Économie : } \\
\hline - PNB global : $1996: 87$ milliards \$ (38 rang mondial) & $\begin{array}{l}\text { France }: 1463,55 \text { milliards } \$ \\
\text { (4' rang mondial) }\end{array}$ \\
\hline - PNB par habitant : $1996: 29002 \$\left(10^{\circ}\right.$ rang mondial $)$ & France : $25190 \$\left(13^{\circ}\right.$ rang mondial $)$ \\
\hline - Parité du pouvoir d'achat : $22750 \$$ (- $15 \%$ du PNB/hab.) & France : $21030 \$(-15,8$ du PNB/hab. $)$ \\
\hline - $\%$ terres cultivées : $7 \%$ & France : $35 \%$ \\
\hline Langue nationale : malais. Langues officielles : malais, tamc & ul, mandarin, anglais. État laïque. \\
\hline
\end{tabular}

\section{Une cité née de la « Méditerranée asiatique »}

4 Située au cœur de la mer de Chine méridionale (Han Bai), l'une des quatre mers de Chine, Singapour est née d'une situation à la fois paradoxale et privilégiée. Dépourvue de ressources naturelles, voire même d'eau potable, cette île exiguë $\left(620 \mathrm{~km}^{2}\right)$, n'a longtemps connu que la fréquentation de pêcheurs malais et de pirates. Pourtant l'île de Temasek (ou Tumasik) se trouvait anciennement déjà placée sur les réseaux d'échanges qui, dès le $\mathrm{X}^{\mathrm{e}}$ siècle, animaient cet espace maritime cerné par les mondes indiens, islamiques, chinois. Les points de rencontre de ces réseaux, véritables interfaces entre les grands ensembles de l'Asie, allaient donner naissance, à la faveur d'un brassage culturel permanent, à des cultures originales et syncrétiques ${ }^{1}$ Une multitude de minorités, souvent mobiles, se trouvaient ainsi projetées sur toutes les parties littorales de la mer de Chine. L'originalité de ces cultures métissées ne fut pas toujours perçue par les Européens, plus fascinés par les royaumes de l'Inde et l'Empire du Milieu. Ce sont pourtant les flux d'échanges plus que les nations qui constituent la réalité de l'Asie du Sud-Est ${ }^{2}$. En cela, la mer de Chine, espace exemplaire de contacts et de continuités culturelles, peut être comparée à la mer Méditerranée.

5 Les Européens, à commencer par les Portugais au XVI siècle, ne firent d'abord que suivre ces routes préexistant à leur arrivée, telle la route de la soie. Ils s'installèrent à proximité des principales intersections, comme Malacca qui fut, dans la zone, le premier port libre, prospère et cosmopolite.

6 La colonisation s'accentuant, se succédèrent dans les détroits et les archipels, Espagnols, Hollandais, Britanniques. Ces derniers prirent progressivement possession de l'île de Temasek, rebaptisée entre temps d'un nom sanscrit, Singhapuri. Devenue colonie de la Couronne en 1867, année de l'ouverture du canal de Suez, et après l'éradication de la piraterie par la Royal $\mathrm{Navy}^{3}$, Singapour connut dès le milieu du XIX siècle un développement rapide détrônant Malacca, au fur et à mesure que les lignes de navigation s'étiraient vers l'Europe ${ }^{4}$. 
7 L'invasion japonaise de 1942 marqua le déclin de la présence britannique mais non de son influence, notamment dans les domaines de l'administration et de l'éducation. Une première étape vers l'affranchissement fut atteinte en 1959 avec l'instauration de l'autonomie dans le cadre de la Fédération de Malaisie.

Cette structure ne résista pas aux tensions avec la Malaisie et à la confrontation armée avec l'Indonésie et prit fin avec l'Indépendance, proclamée le 9 août 1965.

\section{Un modèle de développement fondé sur la diversité culturelle}

9 Dès lors, l'évolution de la cité-État allait tendre vers le développement, selon une formule originale mêlant le libéralisme total et le dirigisme gouvernemental, au prix de soubresauts liés aux périodes de récession (1980-1985), et d'un droit des personnes souvent négligé.

Le développement de Singapour, parti d'une extrême pauvreté génératrice d'émeutes raciales, a puisé ses ressources dans la ténacité et l'esprit d'entreprendre hérités du vaste et permanent brassage des expériences de la mer de Chine. L'évolution s'est ainsi basée sur la coexistence de communautés pourtant bien distinctes.

11 Sur place depuis longtemps, la communauté malaise, en partie sui generis, en partie venue de la péninsule toute proche, en particulier de l'État de Johore de l'autre côté du détroit, représente $15 \%$ de la population avec 415900 personnes $^{5}$. Ces musulmans, souvent d'origine paysanne, ont conservé le mode de vie villageois (kampong), même lorsqu'ils vivent dans des immeubles. On les trouve souvent dans des fonctions cléricales, dans l'enseignement, dans la police et les télécommunications. Le malais demeure la langue nationale.

Les 209400 Indiens (7\% de la population) sont en majorité des paysans originaires de l'Inde du Sud. On retrouve les générations actuelles dans l'administration, le juridique, le journalisme et le petit commerce. La plupart ont conservé l'hindouisme comme religion, vivant à proximité des centaines de temples du quartier Little India. Le tamoul est l'une des quatre langues officielles de Singapour.

La communauté chinoise, forte de 2269600 personnes est la plus nombreuse (75\% de la population). Implantée dans les détroits dès le XVI ${ }^{\mathrm{e}}$ siècle, à l'époque où les premières jonques longeaient les côtes et remontaient les rivières pour commercer, ses effectifs ont rapidement augmenté avec la colonisation britannique. Venus de toutes les régions de la Chine, parlant par conséquent des dialectes différents, partagés entre confucianisme, bouddhisme, taoïsme, islam, christianisme, les Chinois ne constituent pas une communauté homogène $e^{6}$. La plupart d'entre eux ont rompu les attaches avec la terre d'origine depuis plusieurs générations. La majorité ne parle pas le mandarin. Néanmoins, les rites et les croyances de base (le culte des ancêtres, la géomancie), conservent une certaine faveur auprès des jeunes générations pourtant acquises au mode de vie occidental. Les Chinois dominent particulièrement la sphère politique, le commerce régional et international. Le mandarin, le malais, le tamoul constituent avec l'anglais, langue de la gestion et de la technologie, les quatre langues officielles de Singapour. On ajoutera, comme langue vernaculaire, le singlish (Singaporean English), pidgin dérivé de l'anglais et du malais, couramment parlé dans la rue. 
14 Auprès des communautés majoritaires et reconnues officiellement comme telles, le métissage sino-malais a donné naissance à la catégorie des Peranakans. Ces babas (les hommes) et ces nonyas (les femmes) possèdent leur propre culture et leur propre dialecte. D'autres communautés plus minoritaires (Européens, Japonais, Sri-Lankais, etc.), complètent ce paysage pluriethnique caractéristique de la société Singapourienne.

C'est à partir de ce puzzle que la cité-État, fondée en 1965, a commencé de construire une nation. Sur cette terre, démunie de ressources naturelles (à part l'agriculture, l'élevage et la pêche), il est très vite apparu que les ressources humaines les plus diversifiées soientelles, constituaient la vraie richesse. Pour les développer, l'État s'est doté d'un système éducatif capable de promouvoir une culture de l'excellence et de répondre à la gageure du multiculturalisme.

\section{Trois communautés, un seul système}

16 Le système éducatif s'est engagé, à partir des années 1970, non sans autoritarisme politique, dans la voie du développement rapide d'une économie fondée sur l'ouverture sans restriction à tous les partenaires et investisseurs possible. Il est très vite apparu que ce développement gagnait en efficacité à s'appuyer sur les réseaux traditionnels existants, c'est-à-dire à faire fond sur les différentes communautés en présence. Ainsi, chaque communauté se trouvait associée au renforcement et au développement de ces réseaux en mer de Chine et au-delà, vers l'Europe et les États-Unis. La même démarche a contribué à renforcer l'identité nationale, voire même à la créer, en assurant un retour vers les valeurs asiatiques (Asian values), après un siècle de colonisation ${ }^{7}$.

Le programme appelé New Éducation System (NES), mis en œuvre à partir de 1979, s'est donc trouvé associé étroitement à la construction de la jeune nation pluriraciale. La perspective du NES est résolument plurilinguistique et multiculturelle, rendant obligatoire le bilinguisme, dès la première année de l'enseignement primaire ( 6 ans) et pour la durée de l'éducation obligatoire de dix années, à travers l'enseignement de la langue maternelle (mother tongue) et de l'anglais traité comme working language. Par langue maternelle, il faut entendre le choix de l'une des langues des trois communautés majoritaires : tamoul, malais ou mandarin. La généralisation du NES à partir de 1990 s'est accompagnée d'une véritable mobilisation des forces éducatives du pays ainsi que d'une forte revalorisation des salaires, actuellement supérieurs aux salaires européens.

Dans l'enseignement primaire, l'enseignement des langues auquel s'ajoute celui des mathématiques, représente $80 \%$ du temps scolaire. Cet enseignement des langues, depuis 1991, introduit dès la cinquième année du primaire, une première sélection en trois filières suivant les niveaux ${ }^{8}$ :

- la filière EM1, ouverte aux élèves d'un bon niveau linguistique, offre le mandarin, le tamoul ou le malais à un niveau supérieur de même que l'anglais, les mathématiques et les sciences ;

- la filière EM2 offre un niveau moyen pour les mêmes langues et disciplines ;

- la filière EM3 offre un niveau de base complété par un renforcement en mathématiques et en anglais.

19 Un enseignement des cultures et des coutumes accompagne l'ensemble, à l'exception de l'enseignement religieux qui est banni de l'enseignement public, c'est-à-dire du primaire et du secondaire. Le choix de la laïcité s'est en effet imposé à l'État de Singapour comme gage de la paix sociale. La tolérance est vécue au quotidien de manière très concrète dans 
les établissements. Il suffit de voir fonctionner à l'heure des repas les six ou huit cantines différentes proposant les plats traditionnels de chaque communauté pour s'en convaincre.

Depuis 1993, la fin de l'enseignement primaire, à 11 ans donc, est sanctionné par le Primary School Leaving Examination (PSLE) qui détermine l'orientation quasi définitive dans le secondaire, orientation dont les parents sont seulement « informés ». On retrouve, à la base de l'orientation, l'importance accordée aux langues.

Trois voies de chacune quatre années s'offrent alors aux élèves suivant leurs compétences :

- le cursus "Special», avec un niveau supérieur en langue maternelle (malais, mandarin ou tamoul) et en anglais qui se conclut par le Singapore Cambridge General Certificate of Education Ordinary (CGE O-level). Cet examen ouvre la porte des centres pré-universitaires, des junior colleges de même que la voie vers le GCE A-level (Advanced) et les enseignements universitaires;

- le cursus «Express » qui comporte les mêmes disciplines mais à un niveau moyen, avec les mêmes possibilités ;

- le cursus « Normal », divisé en deux filières, l'une, Academic, qui débouche sur le GCE N-level (Normal) et le supérieur technologique (instituts technologiques, polytechnics) et l'autre filière, Technical, qui mène à l'apprentissage. Cette dernière filière qui comprend obligatoirement, outre la langue maternelle et l'anglais, des mathématiques et des applications informatiques, représente environ $20 \%$ de l'effectif de chaque cohorte.

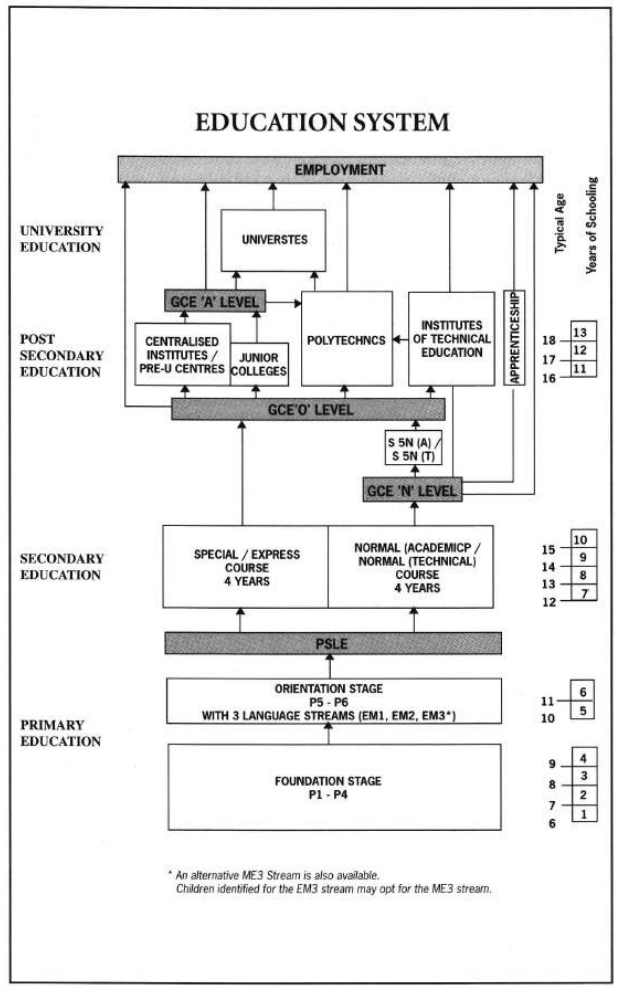

22 Au noyau dur de la scolarité obligatoire, langues maternelles-anglais-mathématiques, s'adjoignent progressivement l'enseignement moral (en l'absence d'enseignement religieux), la musique, l'éducation physique ${ }^{8}$. Des options facultatives concernent la littérature et la civilisation anglaises ainsi que la troisième langue (français, allemand, japonais ou chinois). 
23 À partir de 1999, la suppression des deux mi-temps de travail au profit d'une seule session par jour, libérera du temps libre et des moyens en personnel afin de généraliser le programme de renforcement en langues appelé Language Elective Programme (LEP) qui comporte des séjours d'immersion à l'étranger.

24 L'enseignement privé lui-même (Independant Schools), qui bénéficie d'une liberté totale quant à la définition des cursus, a pour seule obligation de se conformer à l'orientation politique du bilinguisme et du multiculturalisme.

Le fonctionnement du système éducatif est impulsé et supervisé par un corps d'inspection dont la mission est de maintenir le niveau d'excellence dans les établissements et de résoudre les problèmes au fur et à mesure qu'ils apparaissent. C'est ainsi que le bilinguisme pose un certain nombre de difficultés culturelles. En effet, les valeurs véhiculées par l'une et l'autre langue peuvent correspondre à des représentations très différentes du monde. Ces représentations peuvent certes se compléter. Elles peuvent parfois entrer en contradiction. ${ }^{9}$

26 En outre, les trois langues «maternelles» (mother tongues), fréquemment, ne correspondent plus aux langues parlées dans les familles, ni dans la rue. C'est à l'apprentissage d'une véritable langue étrangère que l'élève est le plus souvent confronté.

27 Enfin, ajoutons que les trois mother tongues n'ont pas le même statut socio-économique. Il est évident que le statut de langue internationale du mandarin pèse d'un grand poids dans la détermination des filières d'excellence.

28 Néanmoins, on comprend que le niveau en langues des étudiants qui terminent leur cursus secondaire oscille entre bon et excellent, quelle que soit l'orientation retenue, arts, sciences, technologie ou commerce. On comprend aussi que, devenu productif, le jeune Singapourien n'éprouve aucune difficulté à s'intégrer dans les milieux du travail asiatiques ou occidentaux.

29 Enfin, la stricte égalité des communautés autant que la reconnaissance de leurs différences contribuent, avec le haut niveau d'éducation, à la cohésion sociale et nationale.

On peut raisonnablement mettre au compte de cette éducation pétrie de multiculturalisme et d'exigence, la réussite globale de la formation.

L'adaptation constante aux fluctuations de l'économie mondiale que les décideurs de Singapour ne cessent d'anticiper, a contraint le système éducatif au cours des trente années qui ont suivi l'indépendance à d'incessants et parfois radicaux changements d'orientation. Cette ouverture permanente et cette sensibilité extrême aux variations économiques comportent aussi les germes d'une vulnérabilité que la moindre récession peut rendre catastrophique. Seule la solidité et la souplesse des réseaux, la cohérence et la flexibilité du système éducatif, rendent possibles ces brusques « coups de barre » qui réorientent en quelques semaines tout un secteur d'activité.

Cette réussite, sans équivalent dans l'histoire, sera totale lorsque la cité-État étendra les principes de tolérance à la vie politique et sociale tout entière, permettant un libre exercice de la démocratie et des droits individuels. 


\section{NOTES}

1. Denys Lombard, Le Carrefour Javanais, Paris, EPHES.

2. Charles Fischer, South East Asia, a Social, Economic and Political Geography, London - New.

3. La piraterie a réapparu à partir de 1990, suivant toujours les mêmes réseaux.

4. Les flux en mer de Chine sont actuellement au centre d'enjeux économiques (rivalité de Singapour et de Hong Kong) et militaires particulièrement aigus.

5. Recensement de 1994.

6. Victor Purcell, The Chinese in Malaya, London, Oxford University Press, 1967.

7. Education System at a Glance, Ministry of Education, Singapore, May 1994

8. Les sports de compétition sont importants et font l'objet d'une participation obligatoire au titre des ECA (activités extra-curriculaires). Le Festival de la jeunesse de l'Asie du Sud-Est de l'ASEAN est le couronnement de ces activités.

9. Le Thanh Khoi, L'éducation : cultures et sociétés, Paris, Publications de la Sorbonne, 1991.

\section{RÉSUMÉS}

La réussite présente de Singapour, société multiraciale, dépend en grande partie d'un système d'éducation qui a su intégrer la dimension multiculturelle et pluri-linguistique pour en faire un atout socio-économique majeur: l'excellent niveau en langues des étudiants favorise leur insertion dans tous les milieux, tout comme la stricte égalité des communautés garantit la cohésion nationale.

\section{INDEX}

Index géographique : Singapour

Mots-clés : système scolaire, enseignement des langues, diversité linguistique, pluralisme culturel, apprentissage des langues

\section{AUTEUR}

\section{PIERRE-LOUIS GAUTHIER}

Inspecteur d'académie honoraire, France 